\title{
Pelfings $\mathfrak{W}$ erke
}

umfaffen in biefer burd 5 bramatifase Jugenbarbeiten vermełhrten $\mathfrak{A}$ usigabe 6 Bänbe, unb fojten:

Ifytr. 1. 10. $\mathfrak{M g r}$. pber $\mathfrak{f r} .2 .20 \mathrm{fr}$.

Dieje 2 fusgabe enthält in folgenber (Eintheilung:

Band I. Bsebidite. Fakeln. Der junge Bgelehrte. Die Suben. Der Dilognn. Der Freigeift. Fauft. Der Ediak. Damon. Serther. Die alte Suugfer.

"II. Bjilotas. Míp Sara Sampjon. Minna von Barn= belm. Emilia Balotti. Nathan ser $\mathfrak{B}$ Bije.

"III. Eophofleb. Eaofoon. Erzieb̧ung bes Menfidenge= gefdledte. Briefe antiquarifjen Эnhalte.

"IV. 5̧amburgifhe Dramaturgie.

- V. Babemecum für S. (S. Rarge. Wic bie Alten ben Tob gebilbct. Erntt uno $\mathfrak{F a l f}$. Nod) nähere Beridjtigutg bes Märcjens von 1000 Dutatrn. Fragmente. Rettungen

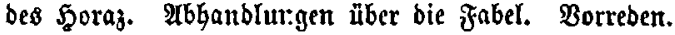
2tuB ben Briefen, bic neucpite Siteratur betreffento.

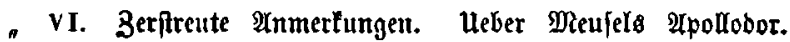
Bom allter ber Delmalerei. Bur Gejdjidjte mo Rite= ratur. Briefe aus bem zmeiten Theil ber Sdriften. Theologifde Streitfdriften. 



\section{$\mathfrak{A e} \int \mathfrak{i t u g s} \mathfrak{W}_{\mathfrak{e r k}}$}

Eriter Banb.

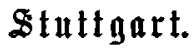

(5). S. (5)

1873. 
Drah von Doerner Gomy. itt Stuttgart. 